\title{
The 0.1-200 keV spectrum of the blazar PKS 2005-489 during an active state
}

\author{
G. Tagliaferri ${ }^{1}$, G. Ghisellini ${ }^{1}$, P. Giommi ${ }^{2}$, A. Celotti ${ }^{3}$, M. Chiaberge ${ }^{3}$, L. Chiappetti ${ }^{4}$, I. S. Glass ${ }^{5}$, \\ L. Maraschi ${ }^{6}$, F. Tavecchio ${ }^{6}$, A. Treves ${ }^{7}$, and A. Wolter 6 \\ 1 Osservatorio Astronomico di Brera, Via Bianchi 46, 23807 Merate, Italy \\ 2 BeppoSAX Science Data Center, ASI, Via Corcolle, 19, 00131 Roma, Italy \\ 3 SISSA/ISAS, via Beirut 2-4, 34014 Trieste, Italy \\ 4 Istituto di Fisica Cosmica G.Occhialini, CNR, Via Bassini 15, 20133 Milano, Italy \\ 5 South African Astronomical Observatory, PO Box 9, Observatory 7935, South Africa \\ 6 Osservatorio Astronomico di Brera, Via Brera, 28, 20121 Milano, Italy \\ 7 Istituto di Fisica, Università dell'Insubria, Via Lucini 3, 22100 Como, Italy
}

Received 10 November 2000 / Accepted 20 December 2000

\begin{abstract}
The bright BL Lac object PKS 2005-489 was observed by BeppoSAX on November 1-2, 1998, following an active X-ray state detected by RossiXTE. The source, detected between 0.1 and $200 \mathrm{keV}$, was in a very high state with a continuum well fitted by a steepening spectrum due to synchrotron emission only. Our X-ray spectrum is the flattest ever observed for this source. The different $\mathrm{X}$-ray spectral slopes and fluxes, as measured by various satellites, are consistent with relatively little changes of the peak frequency of the synchrotron emission, always located below $10^{17} \mathrm{~Hz}$. We discuss these results in the framework of synchrotron self-Compton models. We found that for the BeppoSAX observation, the synchrotron peak frequency is between $10^{15}$ and $2.510^{16} \mathrm{~Hz}$, depending on the model assumptions.
\end{abstract}

Key words. BL Lacertae objects: general - X-rays: galaxies - BL Lacertae objects: individual: PKS 2005-489

\section{Introduction}

The overall spectral energy distribution (SED) of blazars (BL Lac objects and violently variable quasars) shows (in a $\nu$ vs. $\nu F_{\nu}$ representation) two broad emission peaks: the lower frequency peak is believed to be produced by synchrotron emission, while the higher frequency one is probably due to the inverse Compton process. The location of the synchrotron peak is used to define different classes of blazars: HBL (High frequency peaked blazar, with the maximum power output in the UV or X-ray frequencies) and LBL (Low frequency peaked blazar, peaking in the IR or optical bands) (Giommi \& Padovani 1994). Thus, for LBL sources the X-ray emission is dominated by the inverse Compton component.

The continuum emission of blazars is both highly luminous and rapidly variable and it is probably dominated by emission from a jet moving relativistically at small angles to the line of sight (Blandford \& Rees 1978). Strong evidence for bulk relativistic motion was first provided by multiwavelength studies, later confirmed directly with VLBI observations (Vermeulen \& Cohen 1994). However,

Send offprint requests to: G. Tagliaferri,

e-mail: gtagliaf@merate.ni.astro.it single epoch spectra cannot constrain the models of variability in relativistic jets (e.g. Königl 1989; Ulrich et al. 1997). Since blazars emit over the entire electromagnetic spectrum, a key for understanding blazar variability is the acquisition of several wide band spectra in different luminosity states during major flaring episodes. Coupling spectral and temporal information greatly constrains the jet physics, since different models predict different variability as a function of wavelength. Important progress in this respect has been achieved recently for some of the brightest and best studied blazars, as PKS 2155-304 (Chiappetti et al. 1999; Urry et al. 1997), BL Lac (Bloom et al. 1997), 3C 279 (Wehrle et al. 1998), Mkn 501 (Pian et al. 1998), Mkn 421 (Maraschi et al. 1999; Fossati et al. 2000a,b), ON 231 (Tagliaferri et al. 2000). We successfully used the BeppoSAX satellite (Boella et al. 1997a) to perform observations of blazars that were known to be in a high state from observations carried out both in other bands (mainly optical and $\mathrm{TeV}$ ) and in the $\mathrm{X}$-ray band itself. The good BeppoSAX sensitivity and spectral resolution over a very wide $\mathrm{X}$-ray energy range are ideal to constrain the existing models for the X-ray emission.

PKS 2005-489 $(z=0.071)$ is a bright BL Lac object that belongs to the 1 Jy radio catalog (Stickel et al. 1991). 


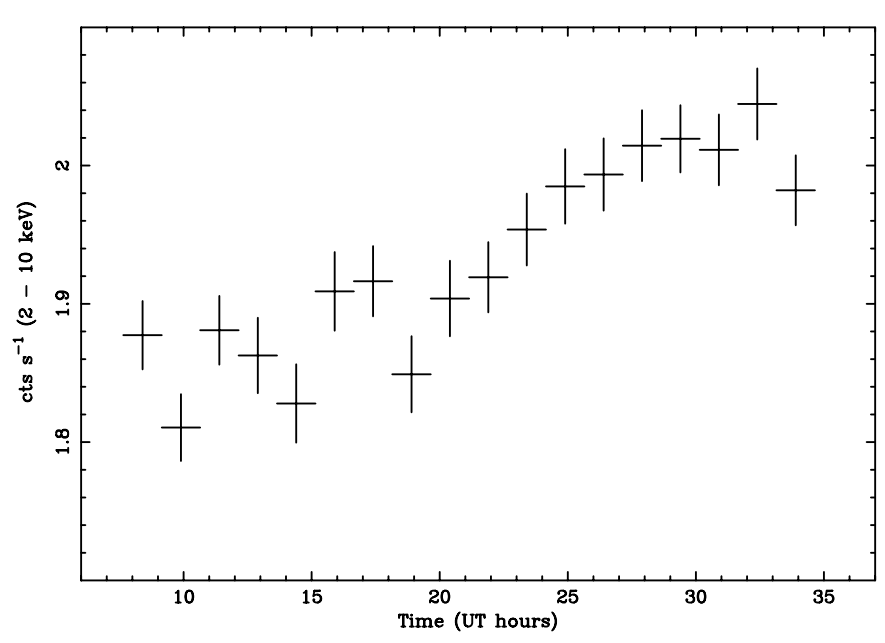

Fig. 1. The 2-10 keV light curve of PKS 2005-489 during the BeppoSAX observation of 1-2 November, 1998. The bin time is of $5400 \mathrm{~s}$

Its broad band spectrum peaks in the UV- soft X-ray bands making it an HBL type of source. It is one of the few extragalactic objects seen in the EUV band (Marshall et al. 1995) and it has been detected in the $\gamma$-ray band by EGRET (von Montigny et al. 1995). In the X-ray band it has been observed 5 times with EXOSAT, showing large flux variations correlated with changes in the spectrum (harder spectrum with increasing flux) (Giommi et al. 1990; Sambruna et al. 1994a,b). These results are also confirmed by two ROSAT observations, which also show a steep $0.2-2.0 \mathrm{keV}$ spectrum, with photon index of about 3 (Sambruna et al. 1995). In September 1996, PKS 2005-489 has been observed by BeppoSAX, that detected the source in a rather bright state, with a flatter $2-10 \mathrm{keV}$ photon index of 2.3 (Padovani et al. 1998, 2000). Finally, a monitoring campaign has been performed in the period October-December 1998 with RossiXTE and the source has been detected up to $40 \mathrm{keV}$ (Perlman et al. 1999). During this campaign the source underwent a strong flare, with a peak to quiescent $2-10 \mathrm{keV}$ flux variation of a factor of 8 (30 with respect to the ROSAT fluxes). This event essentially lasted for all the RossiXTE campaign, peaking on 10 November, thus the source was in a high state for at least three months (Perlman et al. 1999). During the week of 18-25 October, a flare alert was issued by the all-sky monitor (ASM) team (Remillard 1998). This triggered our ToO observation and PKS 2005-489 was observed with BeppoSAX in November 1-2, 1998.

In this paper we present and discuss the results of this BeppoSAX observation together with some simultaneous infrared data.

\section{X-ray observations}

\subsection{Observations and data reduction}

The BeppoSAX satellite is an Italian mission with the participation of The Netherlands Agency for Aerospace Programs (NIVR) and the Space Science Department of the European Space Agency (SSD-ESA). It carries on board four Narrow Field Instruments (NFI) pointing in the same direction and covering a very large energy range from 0.1 to $300 \mathrm{keV}$ (Boella et al. 1997a). Two of the four instruments have imaging capability, the Low Energy Concentrator Spectrometer (LECS), sensitive in the range $0.1-10 \mathrm{keV}$ (Parmar et al. 1997), and the three Medium Energy Concentrator Spectrometers (MECS) sensitive in the range 1.3-10 keV (Boella et al. 1997b). The LECS and three MECS detectors are all Gas Scintillation Proportional Counters and are at the focus of four identical grazing incidence $\mathrm{X}$-ray telescopes. The other two are passively collimated instruments: the High Pressure Proportional Counter (HPGSPC), sensitive in the range $4-120 \mathrm{keV}$ (Manzo et al. 1997) and the Phoswich Detector System (PDS), sensitive in the range 13-300 keV (Frontera et al. 1997). For a full description of the BeppoSAX mission see Boella et al. (1997a).

The data analysis for the LECS and MECS instruments was based on the linearized, cleaned event files obtained from the online archive (Giommi \& Fiore 1998). Light curves and spectra were accumulated with the FTOOLS package (v. 4.0), using an extraction region of 8.5 and 4 arcmin radius for the LECS and MECS, respectively. At low energies the LECS has a broader Point Spread Function (PSF) than the MECS, while above $2 \mathrm{keV}$ the PSFs are similar. The adopted regions provide more than $90 \%$ of the source counts at all energies both for the LECS and MECS.

As expected, PKS 2005-489 was detected in a high state in the $\mathrm{X}$-ray band. However, our observation occurred about one week before the source reached its maximum as monitored by RossiXTE (Perlman et al. 1999). Some flux variability of the order of $10 \%$ on a timescale of about 10 hours was detected during our observations in the $2-10 \mathrm{keV}$ energy band (see Fig. 1). No similar flux variability was detected below $1 \mathrm{keV}$. Given that the amount of variability is quite small, we extracted only one spectrum for the full observation. This corresponds to a total LECS exposure time of $20395 \mathrm{~s}$ and MECS exposure time of $52478 \mathrm{~s}$. The background files for the spectral analysis were accumulated from the long blank field exposures available from the SDC public ftp site (see Fiore et al. 1999; Parmar et al. 1999).

The HPGSPC and PDS were operated in the customary collimator rocking mode, with each collimator pointing alternatively at the source and at the background for $96 \mathrm{~s}$. The HPSGPC has a single collimator, thus the whole instrument is looking at the target for half of the time and to an offset direction for the other half. The total exposure time in our case was $23258 \mathrm{~s}$. The PDS has two collimators, thus one is always looking at the source and the other one at the background. The total PDS exposure time, for the two collimators, was of about $46.8 \mathrm{ks}$. The background subtracted HPGSPC and PDS spectra were obtained from the standard pipeline analysis carried out at the BeppoSAX Science Data Center. 


\subsection{Spectral analysis}

For the spectral analysis, the LECS data have been considered only in the range $0.1-4 \mathrm{keV}$, due to still some unsolved calibration problems at higher energies. To fit the LECS, MECS, HPGSPC and PDS spectra together, one has to introduce constant rescaling factors to account for uncertainties in the inter-calibration of the instruments. The acceptable values for these constants are in the range $0.7-1.0$ for the LECS and in the range $0.77-0.93$ for the PDS, with respect to the MECS. The HPGSPC constant is normally in the range 0.9-1 (Fiore et al. 1999). The spectral analysis was performed with the XSPEC 10.0 package.

In the fitting procedure we first considered a single power law model plus absorption with the column density fixed at the Galactic value $N_{\mathrm{H}}=4.210^{20} \mathrm{~cm}^{-2}$. This model does not give a good fit to the data yielding a reduced $\chi_{\mathrm{r}}^{2}=2.0$ (214 degree of freedom). The fit improved by allowing $N_{\mathrm{H}}$ to vary (see Table 1 ). The power law fit gives a good representation of the $0.1-200 \mathrm{keV}$ $\mathrm{X}$-ray spectrum, with a relative steep photon spectral in$\operatorname{dex} \alpha=2.18$. This index is slightly flatter than the one measured by RossiXTE $(\alpha=2.37 \pm 0.01$ in the $2-10 \mathrm{keV}$ energy band) in the same days (1-2 November, Perlman et al. 1999). Actually, the spectral index determined with BeppoSAX is flatter than all spectral indices obtained with RossiXTE in the period October December, 1998. The flattest RossiXTE spectral index is $\alpha=2.32 \pm 0.015)$ measured on November 6, 1998, a few days before the top of the X-ray flare (Perlman et al. 1999). Moreover, the very high statistics of the LECS and MECS spectra show that from a statistical point of view the power law fit is not acceptable and that a more complex model is required. This is also reflected by the fact that the interstellar absorption, $N_{\mathrm{H}}$, determined in the best fit procedure is higher, at $90 \%$ confidence, than the Galactic value. This implies either the existence of an intrinsic absorption in PKS 2005-489 which seems unlikely, or that we are using a wrong model.

We therefore considered a broken power law model which gave a much better fit, even if we keep the $N_{\mathrm{H}}$ fixed at the Galactic value. The difference between the two spectral indices, although significant, is small, with the break at $\sim 2 \mathrm{keV}$. The spectral index above the break is the same as the single power law, while before the break it is somewhat flatter (see Table 1 and Fig. 2). Of course, this break at $2 \mathrm{keV}$ could not have been detected by RossiXTE. In this fit the LECS, HPGSPC and PDS intercalibration constant factors have also better values (see notes in Table 1). Clearly we are detecting a downward curved spectrum, probably the declining part of the synchrotron emission. Thus, we considered a more physical intrinsically curved spectral model developed by Fossati et al. (2000b). This model is an analytic expression that assumes a continuously changing slope between two asymptotic limits. It is expressed in a form that provides the following parameters $\left(E_{1}, \alpha_{1}, E_{2}, \alpha_{2}, E_{B}, f\right)$, where $E_{1}$ and $E_{2}$ are the two "pivoting energies", while $E_{B}$ and $f$ determine the scale length of the curvature. In order to fit this model to the

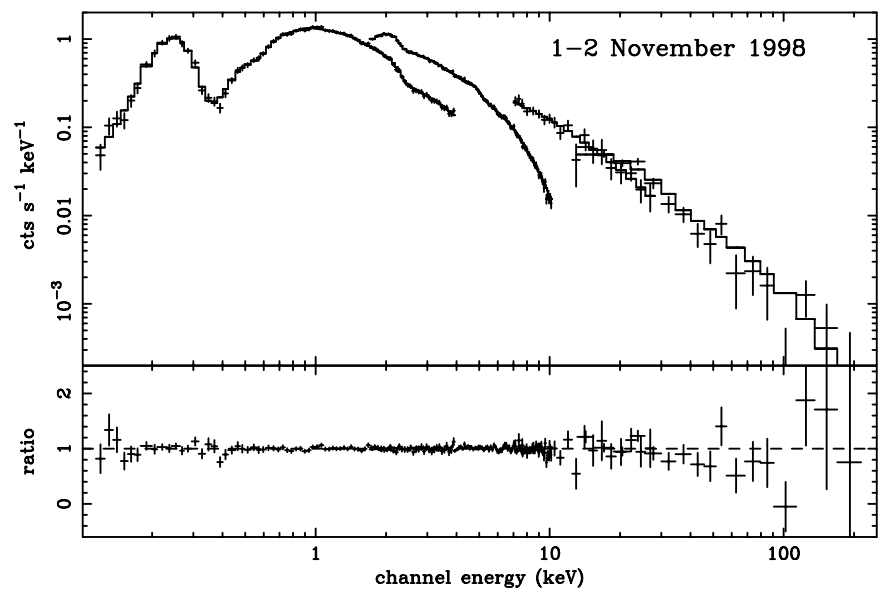

Fig. 2. LECS + MECS + HPGSPC + PDS spectrum observed on 1-2 November 1998, the best fit model is a broken power law model. The $N_{\mathrm{H}}$ was fixed to the Galactic value

data we have to fix one parameter for each of the two pairs $\left(E_{1}, \alpha_{1}\right)$ and $\left(E_{2}, \alpha_{2}\right)$. If we fix the energy we will find the spectral index at that energy, while if we fix the spectral index we will find the energies where it will occur. The latter possibility is very important, because it allows us to find the peak of the synchrotron component, if this is inside our energy band, and to estimate its uncertainty. This can be obtained by setting one spectral index, for instance $\alpha_{1}=1$, so that the best-fit value of $E_{1}$ will give $E_{\text {peak }}$. For more details we refer to the Fossati et al. (2000b) paper. In order to determine the values of the really interesting parameters and their confidence intervals, in the best-fit procedure we kept the absorption column density fixed to the Galactic value and $f$ fixed to the best-fit value $f=4.5$. If we fix the two energies at $E_{1}=0.1$ and $E_{2}=100 \mathrm{keV}$, we find that the respective photon spectral indices are $\alpha_{1}=1.98_{+0.02}^{-0.03}$ and $\alpha_{2}=2.23 \pm 0.02$. We then fixed the photon energy index $\alpha_{1}=2$ in order to find the synchrotron peak and indeed we find that $E_{\text {peak }}=1.15_{+0.15}^{\text {-n.c. }} \mathrm{keV}$. This confirms the presence of a curved spectrum with a synchrotron peak below $\sim 1.5 \mathrm{keV}$. It is in any case remarkable that this curvature from 0.1 up to $100 \mathrm{keV}$ is rather small with respect to other sources. All quoted errors, for the curved model, are calculated for $\Delta \chi^{2}=2.7$ that corresponds to a $90 \%$ confidence range for one parameter of interest.

\section{Infrared observations}

The JHKL observations were made using the MkIII infrared photometer attached to the SAAO $1.9 \mathrm{~m}$ telescope at Sutherland, in July 1997 and in October 1998, the latter was one day before the start of the BeppoSAX observation. The chopper throw was 30 arcsec in a N-S direction and the aperture was 12 arcsec diameter. This aperture contains a large fraction of the galaxy whose H-flux is comparable ( $\sim 20 \%$ lower) to the $\mathrm{H}$-flux of the nucleus (see Kotilainen et al. 1998). The values reported in Table 2 and Fig. 4 are not corrected for the galaxy contribution. 
Table 1. Fit results for a power law and broken power law model

\begin{tabular}{|c|c|c|c|c|c|c|c|}
\hline model $^{\mathrm{a}}$ & $\Gamma_{1}^{\mathrm{b}}$ or $N_{\mathrm{H}}$ & $\Gamma_{2}$ & $\begin{array}{l}\text { break } \\
\text { energy }^{c}\end{array}$ & $\chi_{\mathrm{r}}^{2}$ (d.o.f.) & $\begin{array}{c}F_{[0.1-2 \mathrm{keV}]}^{\mathrm{d}} \\
\operatorname{erg~cm}^{-2} \mathrm{~s}^{-1}\end{array}$ & $\begin{array}{c}F_{[2-10 \mathrm{keV}]} \\
\operatorname{erg~cm}^{-2} \mathrm{~s}^{-1}\end{array}$ & $\begin{array}{c}F_{[10-100 \mathrm{keV}]} \\
\operatorname{erg~cm}^{-2} \mathrm{~s}^{-1}\end{array}$ \\
\hline $\begin{array}{l}\text { power law } \\
\text { broken p. } 1 .\end{array}$ & $\begin{array}{c}\left(4.9_{+0.15}^{-0.15}\right) 10^{20} \\
2.02_{+0.03}^{-0.04}\end{array}$ & $\begin{array}{l}2.18_{+0.02}^{-0.02} \\
2.21_{+0.02}^{-0.02}\end{array}$ & $1.85_{+0.45}^{-0.35}$ & $\begin{array}{c}1.4(213) \\
0.90(212)\end{array}$ & $4.010^{-10}$ & $1.810^{-10}$ & $1.710^{-10}$ \\
\hline
\end{tabular}

${ }^{a}$ we had also a constant to allow for uncertainties in the intercalibration of the instruments (see Sect. 2.2); the best fit values are 1) PL: LECS constant $=0.75$, MECS constant $=1$. frozen, HPGSPC constant $=0.94$, PDS constant $=0.72 ; 2)$ BPL: LECS constant $=0.78$, MECS constant $=1$. frozen, HPGSPC constant $=0.97$, PDS constant $=0.77 ;{ }^{\mathrm{b}}$ photon spectral index; ${ }^{\mathrm{c}}$ energy values in $\mathrm{keV} ;{ }^{\mathrm{d}}$ corrected for the absorption.

Table 2. Infrared observations

\begin{tabular}{llcccc}
\hline & & & & & \\
date & Jul. Date & $J^{\mathrm{a}}$ & $H$ & $K$ & $L$ \\
27 July 1997 & 2450657 & 10.97 & 10.23 & 9.55 & $8.52 \pm 0.03$ \\
& 2450721 & 11.17 & 10.41 & 9.75 & $8.80 \pm 0.08$ \\
& 2450738 & 11.23 & 10.51 & 9.87 & $8.91 \pm 0.05$ \\
& 2450742 & 11.26 & 10.49 & 9.84 & $8.70 \pm 0.07$ \\
30 Oct. 1998 & 2451117.3 & 11.16 & 10.46 & 9.80 & $8.69 \pm 0.04$ \\
\hline
\end{tabular}

a The errors for the $J, H, K$ magnitudes are $\leq 0.03$.

However, on the scale of Fig. 4 this correction is negligible and smaller than the size of the infrared data symbols. The calibration in terms of log flux in $\mathrm{W} \mathrm{m}^{-2} \mathrm{~Hz}^{-1}$ for a zeroth mag star on this system at $J H K L$ are -22.82 , $-23.01,-23.21$ and -23.55 respectively.

As it is usually found for HBL, there is very little change in the slope of the infrared spectrum as the luminosity changes (see Table 2), i.e. the infrared colour of the blazar changes little or not at all. This is due to the fact that the synchrotron peak is at much higher energies. Changes in $K$ of up to four mags have been seen in some blazars with little change in their infrared colours (e.g. Fan 1999; Hagen-Thorn et al. 1994). In the particular case of PKS 2005-489, the $K$ mag changed only by 0.32 mag during the observations, which is relatively little for this class of objects.

\section{Discussion}

\subsection{SED}

We observed PKS 2005-489 while it was in a very high state, aiming at detecting a change in its spectral energy distribution (SED) with respect to previous observations. Very significant changes of the overall SED were in fact detected in other HBL objects, such as MKN 501 (Pian et al. 1998) and 1ES 2344+514 (Giommi et al. 2000): in these objects, during flares, the synchrotron peak frequency increased by $2-3$ orders of magnitudes with respect to quiescent states. These observations provide new conditions that the various models have to reproduce, exploring new range of values for the physical parameters and allowing us to investigate the accelerating mechanisms inside the jets.

In the case of PKS 2005-489, the X-ray data can be fitted equally well by a broken power law and by a continuously steepening model. Although the spectral parameters are not very different, the two models predict a quite different frequency for the peak of the synchrotron emission, as illustrated in the two panels of Fig. 4. In the case of a broken power law in fact, it can be possible to interpret the entire far-IR to hard X-ray SED as due to a single synchrotron component, peaking at $\sim 10^{15} \mathrm{~Hz}$. If, instead, the $\mathrm{X}$-ray data are fitted by the continuously steepening model, then the synchrotron peak is at $\sim 0.1 \mathrm{keV}$, and the IR flux must be produced by another jet component. This uncertainty in locating the synchrotron peak frequency originated the two possible sets of parameters of the homogeneous synchrotron self Compton model by which we can interpret the overall SED, discussed below.

In any case, our data show that the source was in a very high state and that the $0.1-200 \mathrm{keV}$ spectrum is well fitted by a spectrum due to synchrotron emission only. In Fig. 3 we plot an enlargement of the SED in the X-ray spectral region. It can be seen that PKS 2005-489 underwent dramatic flux changes, but, at least above $\sim 1 \mathrm{keV}$, the spectral shape was always steeper than unity, constraining the peak of the synchrotron emission to be at lower energies. Our X-ray spectrum is the flattest ever observed, even considering the many observations by RossiXTE during the same period of our 1998 BeppoSAX observation (Perlman et al. 1999).

\subsection{Limits on magnetic field and particle energies}

The spectral properties of our X-ray data indicate that the dominating radiation process is synchrotron. Therefore the emission is due to the most energetic electrons, suffering rapid radiation energy losses. During our observation the flux variability was modest $(10 \%$ on a timescale of about 10 hours), but EXOSAT, in two occasions, observed flux changes of a factor of about 4 in $4-5$ hours (Giommi et al. 1990). We can then require that the emitting electrons cool radiatively (by synchrotron and inverse Compton) on these timescales, implying a lower 


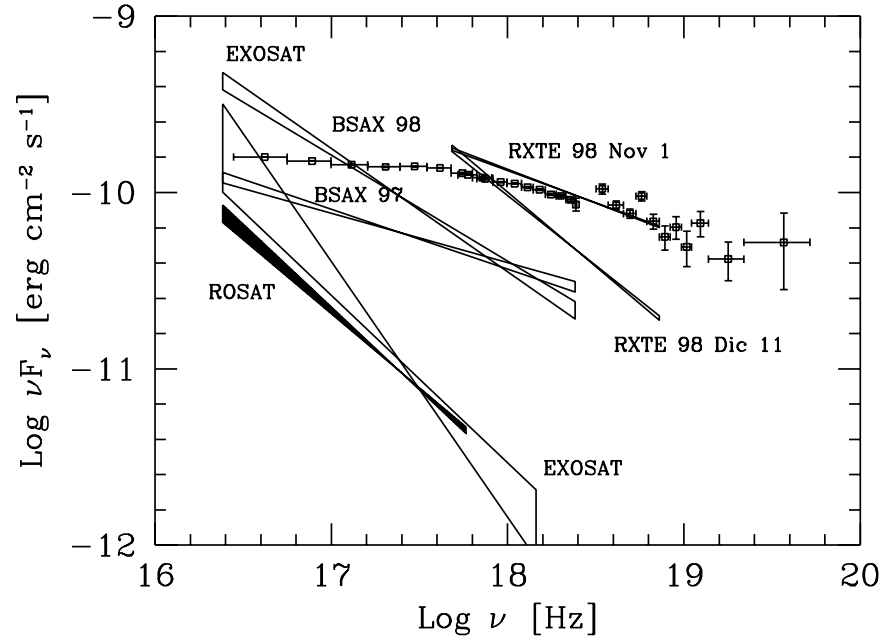

Fig. 3. The X-ray energy spectra of PKS 2005-489 as observed at different epochs by different satellites. Source of data: Sambruna et al. (1994b) (EXOSAT), Comastri et al. (1997) (ROSAT), Perlman et al. (1999) (RossiXTE)

limit on the magnetic field. Assuming equal radiation and magnetic energy densities, using $t_{\mathrm{var}}=4$ hours and $\nu_{x}=110^{17} \mathrm{~Hz}$, we derive $B>0.3 \delta^{-1 / 3}$ Gauss and $\gamma_{x}<310^{5} \delta^{-1 / 3}$. Here $\delta=\left[\Gamma-\sqrt{\Gamma^{2}-1} \cos \theta\right]^{-1}$ is the Doppler beaming factor, where $\theta$ is the viewing angle.

\subsection{Homogeneous SSC model}

We have fitted the SED of PKS 2005-489 with a pure, homogeneous and one zone SSC model, assuming no contribution from the photons produced externally to the jet as seeds for the Compton scattering process. This class of SSC models is completely constrained once the peak energies and their fluxes are known. Unfortunately, in our case only the X-ray and IR data are simultaneous, and the location of both peaks (particularly the Compton one), is somewhat uncertain. However, the $\mathrm{X}$-ray data alone constrain the synchrotron peak to be at or below the low energy end of the BeppoSAX range (i.e. $\leq 0.1 \mathrm{keV}$ ). For the high energy part of the SED we have considered the previous observation by EGRET, resulting in a marginal detection, see Fig. 4, (Lin et al. 1997; von Montigny et al. 1995) and the $\mathrm{TeV}$ upper limit given by CANGAROO (Roberts et al. 1999). These data constrain the Compton peak to be close to $10^{25} \mathrm{~Hz}$.

Given the uncertainty in the location of the synchrotron peak, we have considered two models, shown in the two panels of Fig. 4. The top panel shows the X-ray data fitted by a broken power law model, and in this case it is possible that the simultaneous IR flux is produced in the same region producing the high energy emission. We have then considered a source with size $R=1.510^{16} \mathrm{~cm}$ and a beaming factor $\delta=18$, resulting in a minimum observed variability timescale of $\sim 6$ hours, which is typical for this class of sources, and close to the minimum variability timescale observed in this source by EXOSAT

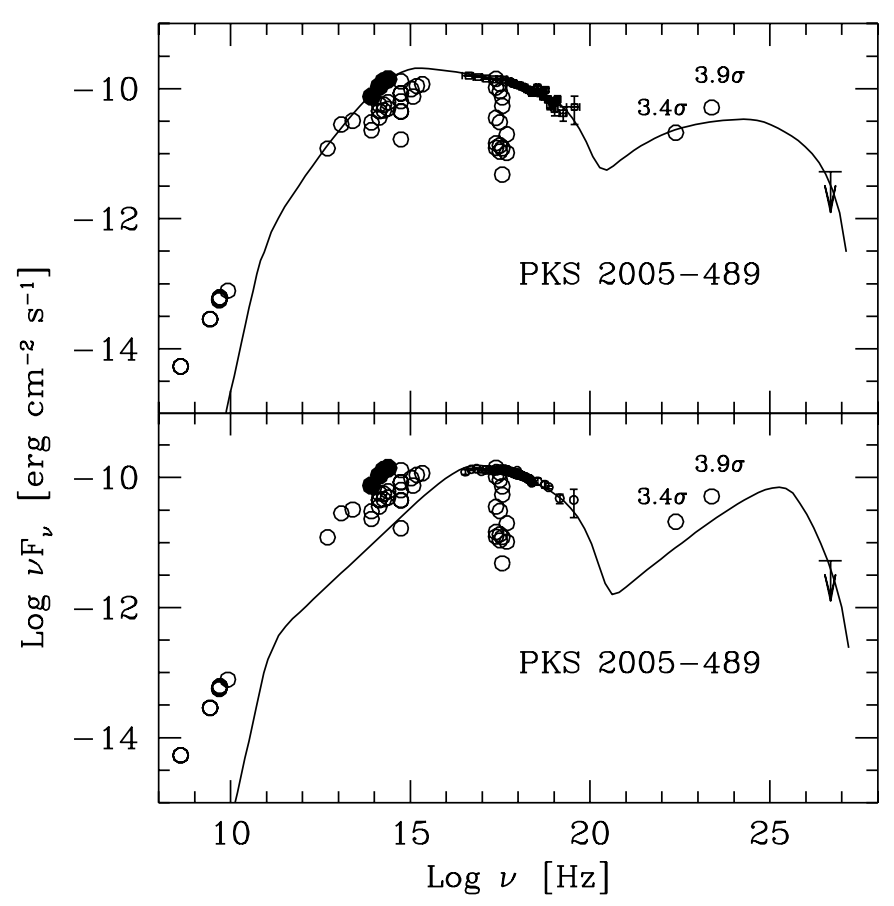

Fig. 4. The SED of PKS 2005-489. Note that only the BeppoSAX and the IR data (filled symbols) are simultaneous. The other data have been taken from the Nasa Extragalactic Database (NED). In the top panel we used for the X-ray spectrum the broken power law best-fit model. In this case the SSC model fits also the IR data. In the bottom panel we used for the X-ray spectrum the curved best-fit model (see text). In this case the IR emission is not due to the same SSC component responsible for the X-ray emission. For both SSC models we have assumed no external photons contributing to the inverse Compton process. The input parameters for the top (bottom) models are: $L_{\text {inj }}^{\prime}=5.510^{41}\left(7.810^{41}\right) \mathrm{erg} \mathrm{s}^{-1}, B=1$ (1) Gauss, $R=1.510^{16}\left(10^{16}\right) \mathrm{cm}, \gamma_{\min }=310^{3}\left(1.510^{4}\right)$, $\gamma_{\max }=10^{6}\left(1.2510^{6}\right), s=2.2(2.3), \delta=18(15)$

(Giommi et al. 1990). With these values of the size and beaming factor, the magnetic field is constrained to be of the order of $B=1$ Gauss, in order to have nearly equal synchrotron and self-Compton luminosities, as observed. The intrinsic power (assumed to be continuously injected throughout the source in the form of relativistic electrons) is required to be $L_{\mathrm{inj}}=5.510^{41} \mathrm{erg} \mathrm{s}^{-1}$. The relativistic electrons are assumed to be injected with a power law energy distribution of slope $s=2.2$ between $\gamma_{\min }=310^{3}$ and $\gamma_{\max }=10^{6}$. The value $s=2.2$ is required to match the slope of the $\mathrm{X}$-ray spectrum. The resulting SSC spectrum is shown as a solid line in the top panel of Fig. 4. It can be seen that this model also agrees with the (admittedly uncertain) flux in the EGRET band, and well describes the entire SED (except for the mm-radio part, which should come from larger jet regions, less affected by synchrotron self-absorption at these frequencies). Being produced by the same region, we expect some variability correlations between the IR and X-ray flux. In particular, if the radiative cooling time at both frequencies is shorter than the light crossing time, then it is the latter timescale 
that is observed in both energy bands. With the adopted parameters, this is indeed the case: at the observed frequency $\nu=310^{14} \mathrm{~Hz}$, the intrinsic cooling time is half the crossing time.

The bottom panel of Fig. 4 shows the X-ray data fitted by the continuously steepening model. In this case the IR flux cannot be produced by the same region emitting the $\mathrm{X}$-rays, resulting in a significantly larger frequency of the synchrotron peak. The adopted parameters of the SSC model are similar to the previous case (see the caption of Fig. 4). The only significant difference is the value of $\gamma_{\text {min }}=1.510^{4}$, now much larger.

In this case we predict/expect that the IR flux has a different variability behavior than the $\mathrm{X}$-ray flux, since it would probably be produced in a larger region of the jet, with a smaller value of the magnetic field, making the cooling time of the IR emitting electrons longer. Furthermore, if the variability is caused by a perturbation of a steady jet propagating from the inner to the outer regions, we expect some delay between the $\mathrm{X}$-ray and the IR flux variations, giving information about the relative distance of the two regions.

Acknowledgements. This research was financially supported by the Italian Space Agency. We thank the BeppoSAX Science Data Center (SDC) for their support in the data analysis. This research made use of the NASA/IPAC Etragalactic Database (NED) which is operated by the Jet Propulsion Laboratory, Caltech, under contract with the National Aeronautics and Space Administration.

\section{References}

Blandford, R. D., \& Rees, M. J. 1978, in Pittsburgh Conference on BL Lac Objects, Pittsburgh, University of Pittsburgh, 328

Bloom, S. D., Bertsch, D. L., Hartman, R. C., et al. 1997, ApJ, 490, L145

Boella, G., Butler, R., Perola, G. C., et al. 1997a, A\&AS, 122, 299

Boella, G., Chiappetti, L., Conti, G., et al. 1997b, A\&AS, 122, 327

Chiappetti, L., Maraschi, L., Tavecchio, F., et al. 1999, ApJ, 521,552

Comastri, A., Fossati, G., Ghisellini, G., \& Molendi, S. 1997, ApJ, 480, 534

Fan, J. H. 1999, MNRAS, 308, 1032

Fiore, F., Guainazzi, M., \& Grandi, P. 1999, Cookbook for NFI BeppoSAX Spectral Analysis v. 1.2, available at www.sdc.asi.it

Fossati, G., Celotti, A., Chiaberge, M., et al. 2000a, ApJ, 541, 153

Fossati, G., Celotti, A., Chiaberge, M., et al. 2000b, ApJ, 541, 166
Frontera, F, Costa, E., Dal Fiume, D., et al. 1997, A\&AS, 122, 357

Giommi, P., Barr, P., Garilli, B., Maccagni, D., \& Pollock, A. M. T. 1990, ApJ, 356, 432

Giommi, P., \& Fiore, F. 1998, in The 5th International Workshop on Data Analysis in Astronomy, Erice, Italy, ed. V. Di Gesù, M. J. B. Duff, A. Heck, M. C. Maccarone, L. Scarsi, \& H. U. Zimmermann (Word Scient. Publ.), 73

Giommi, P., \& Padovani, P. 1994, ApJ, 444, 567

Giommi, P., Padovani, P., \& Perlman, E. 2000, MNRAS, 317, 743

Hagen-Thorn, V. A., Yakoleva, V. A., Takalo, L. O., \& Sillanpää, A. 1994, A\&A, 290, 1

Königl, A. 1989, in BL Lac Objects, ed. L. Maraschi, T. Maccacaro, \& M.-H. Ulrich (Berlin: Springer-Verlag), 321

Kotilainen, J. K., Falomo, R., \& Scarpa, R. 1998, A\&A, 336, 479

Lin, Y. C., et al. 1997, 4th Comp. Symp, AIP Conf. Proc. 410, 1317, ed. C. D. Dermer, M. S. Strickman, \& J. D. Kurfess

Manzo, G., Giarrusso, S., Santangelo, A., et al. 1997, A\&AS, 122,341

Maraschi, L., Fossati, G., Tavecchio, F., et al. 1999, ApJ, 526, L81

von Montigny, C., Bertsch, D. L., Chiang, J., et al. 1995, ApJ, 440,525

Padovani, P., Costamante, L., Giommi, P., et al. 2000, in preparation

Padovani, P., Giommi, P., Comastri, A., et al. 1998, Nucl. Phys. B (Proc. Suppl.) 69, 431

Parmar, A. N., Oosterbroek, T., Orr, A., Guainazzi, M., Shane, N., Freyberg, M. J., Ricci, D., \& Malizia, A. 1999, A\&AS, 136,407

Parmar, A. N., Martin, D. D. E., Baddaz, M., et al. 1997, A\&AS, 122, 309

Perlman, E. S., Madejski, G., Stocke, J. T., \& Rector, T. A. 1999, ApJ, 523, L11

Pian, E., Vacanti, G., Tagliaferri, G., et al. 1998, ApJ, 492, L17

Remillard, R. 1998, IAU Circ., 7041

Roberts, M. D., McGee, P., Dazeley, S. A., et al. 1999, A\&A, 343,691

Sambruna, R. M., Barr, P., Giommi, P. Maraschi, L., Tagliaferri, G., \& Treves, A. 1994a, ApJ, 434, 468

Sambruna, R. M., Barr, P., Giommi, P. Maraschi, L., Tagliaferri, G., \& Treves, A. 1994b, ApJS, 95, 371

Sambruna, R. M., Urry, C. M., Ghisellini, G., \& Maraschi, L. 1995, ApJ, 449, 567

Tagliaferri, G., Ghisellini, G., Giommi, P., et al. 2000, A\&A, 354,431

Ulrich, M.-H., Maraschi, L., \& Urry, C. M. 1997, ARA\&A, 35, 445

Urry, C. M., Treves, A., Maraschi, L., et al. 1997, ApJ, 486, 799

Vermeulen, R. C., \& Cohen, M. H. 1994, ApJ, 430, 467

von Montigny, C., Bertsch, D. L., Chiang, J., et al. 1995, ApJ, 440,525

Wehrle, A. E., Pian, E., Urry, C. M., et al. 1998, ApJ, 497, 178 